\title{
Predicting Bevirimat resistance of HIV-1 from genotype
}

Dominik Heider $^{1 *}$, Jens Verheyen ${ }^{2}$, Daniel Hoffmann ${ }^{1}$

\begin{abstract}
Background: Maturation inhibitors are a new class of antiretroviral drugs. Bevirimat (BVM) was the first substance in this class of inhibitors entering clinical trials. While the inhibitory function of BVM is well established, the molecular mechanisms of action and resistance are not well understood. It is known that mutations in the regions CS p24/p2 and p2 can cause phenotypic resistance to BVM. We have investigated a set of p24/p2 sequences of HIV-1 of known phenotypic resistance to BVM to test whether BVM resistance can be predicted from sequence, and to identify possible molecular mechanisms of BVM resistance in HIV-1.

Results: We used artificial neural networks and random forests with different descriptors for the prediction of BVM resistance. Random forests with hydrophobicity as descriptor performed best and classified the sequences with an area under the Receiver Operating Characteristics (ROC) curve of $0.93 \pm 0.001$. For the collected data we find that p2 sequence positions 369 to 376 have the highest impact on resistance, with positions 370 and 372 being particularly important. These findings are in partial agreement with other recent studies. Apart from the complex machine learning models we derived a number of simple rules that predict BVM resistance from sequence with surprising accuracy. According to computational predictions based on the data set used, cleavage sites are usually not shifted by resistance mutations. However, we found that resistance mutations could shorten and weaken the $\alpha$-helix in p2, which hints at a possible resistance mechanism.

Conclusions: We found that BVM resistance of HIV-1 can be predicted well from the sequence of the p2 peptide, which may prove useful for personalized therapy if maturation inhibitors reach clinical practice. Results of secondary structure analysis are compatible with a possible route to BVM resistance in which mutations weaken a six-helix bundle discovered in recent experiments, and thus ease Gag cleavage by the retroviral protease.
\end{abstract}

\section{Background}

\section{HIV and Bevirimat}

Bevirimat (BVM) [1] belongs to a new class of anti-HIV substances that inhibit maturation of virus particles by preventing cleavage of precursor polyprotein by the retroviral protease (PR). BVM prevents the final cleavage of precursor protein p25 to p24 and p2, hence p25 proteins are accumulating in the immature virions. These immature viral particles are not capable of transforming to an infectious stage, and the viral replication cycle is interrupted. A first set of mutations conferring resistance to BVM were found in selection experiments with BVM and were located at CS p24/p2 [1-4]. In clinical phase II trials, polymorphisms in the QVT-motif of $\mathrm{p} 2$

\footnotetext{
*Correspondence: dominik.heider@uni-due.de

'Department of Bioinformatics, Center of Medical Biotechnology, University of Duisburg-Essen, Universitaetsstr. 2, 45117 Essen, Germany
}

were found to prevent antiretroviral activity of BVM and were extensively studied in phenotypic resistance assays [5-7].

\section{Machine learning}

The notion of a resistance mutation is often useful as a first, simple approximation to describe relations between point mutations and resistance phenotypes. However, it is often observed that the more data become available the more complex are the relations between genotype and phenotype that show up. For instance, it has been observed that mutations in the QVT motif (wild type sequence 369-371) are preferentially associated with resistance to BVM [8]. However, as the data analyzed in the current study shows, the same set of mutations of QVT to QAS can be associated with BVM resistance [5] or susceptibility [6], depending on the mutational 
background. Machine learning methods are built to cope with such complex associations.

There are several machine learning methods that have been successfully employed to this end, e.g. rule-based methods [9], decision trees [10,11], support vector machines [12], random forests (RFs) [13], or artificial neural networks (ANNs) [14-16].

ANNs are universal approximators that can be used to solve non-linear classification problems; they are prone to overtraining if not properly set up $[17,18]$. RFs are also excellent non-linear models, and in general perform better than single decision trees (DTs) [19]. They are less easily interpretable than DTs, although they provide variable importance measures [20]. In contrast, rule based systems yield rules that are well intelligible, but often classify not optimally [21,22].

\section{Methods \\ Data}

Sequences of the p24/p2 region of 45 strains of HIV-1 with susceptibility or intermediate resistance to BVM (here defined as $I C_{50} \leq 10$ ) were used, and 110 sequences of resistant strains $\left(I C_{50}>10\right)$. The phenotype was determined in experiments in which HIV-1 was cultured in the presence of increasing concentrations of BVM. The concentration of BVM inhibiting $50 \%$ of viral replication compared to cell culture experiments without $\mathrm{BVM}$ is defined as $I C_{50}(50 \%$ inhibitor concentration). In general, drug resistance means reduced inhibition of viral replication by antiretroviral drugs, resulting in increased $I C_{50}$ values. The $I C_{50}$ values of the drug resistant isolates and HIV wild type are used to calculate resistance factors

$$
\frac{I C_{50}(\mathrm{BVM} \text { concentration for resistant strain })}{I_{50}(\mathrm{BVM} \text { concentration for wild type })}
$$

a standardized measure of HIV drug resistance. The cut-off value of the resistance factor used to define the classes "resistant to BVM" and "susceptible to BVM" was previously derived from data obtained in phase II clinical trials with BVM correlating phenotypic resistance and clinical response [6,7].

All data were collected from several studies that have investigated polymorphisms in $\mathrm{p} 2$, especially in its C-terminal half [1,5-7] (see additional file 1 for complete set). Duplicated sequences in each class were removed prior to analysis.

\section{Multiple Sequence Alignment}

Multiple sequence alignments of the sequences were produced with clustalw [23], t-coffee [24], muscle [25], and prank [26]. Clustalw and muscle gave very compact alignments with a width of 21 columns and most rows free of gaps. The alignment from $\mathrm{t}$-coffee was wider by one column, and the prank alignment much wider with 36 columns. Since clustalw and muscle gave similar alignments, and the prank alignment led to a relatively poor predictive performance, we restrict ourselves in the following to reporting results based on the output of clustalw and t-coffee (see additional files 2 and 3 ).

\section{Descriptor set}

It is often helpful to analyze not the sequences of amino acids as strings of characters, but to associate with each amino acid a numerical "descriptor" value, for instance a value that captures a physico-chemical property of this amino acid. Recently, it has been shown that the descriptor set is the most critical element in classification $[27,28]$, and that physico-chemical descriptors outperform simpler descriptors [29]. In our search for a method with maximum predictive power we tested several numerical descriptors, including hydrophobicity values of Kyte and Doolittle [30], molecular weight, isoelectric point (IEP) and $\mathrm{pKa}$ values for each amino acid. Moreover, we used the predicted probability for cleavage by HIV protease as a descriptor [31]. The numerical descriptor values for gaps from the multiple sequence alignment are undefined $a$ priori. We therefore tested three values for gaps, namely $0,-1$ and an interpolated value (mean of the two amino acid descriptor values on both sides of gap). In the case of 0 and interpolated values for gaps the descriptor values of the amino acids were normalized to the interval $[-1,1]$, and in the case of -1 for a gap they were normalized to $[0,1]$. Apart from using numerical descriptors, we also trained an RF with the multiply aligned p2 sequences using as factors the single letter codes of the amino acids and "-" for gaps.

\section{Neural Networks}

We used a Java implementation http://www.heatonresearch.com/encog of neural networks with one hidden layer and three to seven hidden neurons. Resilient propagation (Rprop) was applied as a learning rule [32]. We used the identity function as activation function for the input layer and the logistic function for the hidden and output layer, respectively. We have used the logistic function because it has been shown in recent studies that it leads to a better generalization ability $[33,34]$. The weights of the ANNs were initiated by applying the Nguyen-Widrow-method [35]. Stop-training was performed in order to avoid overfitting of the neural networks [36].

\section{Random Forests}

As an alternative to ANNs we trained Random Forests (RFs) [20] for the prediction of BVM resistance, using the implementation in the randomForest package of $\mathrm{R}$ [37]. In our application each RF consisted of 2000 randomly and independently grown decision trees. When using the trained RF for prediction, an unseen sequence was assigned to the resistance class voted for by at least $50 \%$ of 
the trees. The importance of each variable, i.e. sequence position, for the correct classification can be assessed by determining the increase in misclassification rate due to leaving this variable [20]. Furthermore, we used the rpart package of $\mathrm{R}$ [37] to create single decision trees.

\section{Rule-based systems}

We used the rule-based algorithms JRip [38] and PART [39] as implemented in R [37].

\section{Cross-validation}

All machine learning methods were validated using 100fold leave-one-out [40] validation to assess for the different machine learning methods the mean prediction sensitivity, specificity, and accuracy (see formulas below) and the ability to generalize to unseen sequences. In addition to this cross-validation, we report for RFs an out-of-bag (OOB) error, an upper limit of the classification error [20].

For each test in the cross-validation, the sensitivity, specificity, and accuracy were calculated according to:

$$
\begin{gathered}
\text { sensitivity }=\frac{T P}{T P+F N} \\
\text { specificity }=\frac{T N}{T N+F P} \\
\text { accuracy }=\frac{T P+T N}{T P+T N+F P+F N} .
\end{gathered}
$$

with true positives $T P$, false positives $F P$, false negatives $F N$ and true negatives $T N$. Figure 1 shows sensitivities and specificities as ROC curves (Receiver Operating Characteristics) [41] for the non-discrete methods in our study. Table 1 gives the corresponding areas under the curve (AUC). ROC curves were drawn with R-package ROCR [42].

\section{Structure and cleavage-site prediction}

Secondary structures of all p2 sequences of 20 or more residues were predicted using JPred [43]. Based on statistical evidence, the secondary structure predictions did also yield a reliability index from 0 (unreliable) through 9 (highly reliable) for each residue being in a predicted secondary structure state.

HIV protease cleavage sites for all p2 sequences were predicted with HIVcleave [31] based on earlier work by Chou et al. [44].

\section{Statistical comparison}

All models were compared by applying Wilcoxon Signed-Rank test [45] on the AUC distributions from the 100-fold leave-one-out cross-validation runs [46]. The null hypothesis was that there are no differences between the compared classifiers.

\section{Results and Discussion}

\section{Prediction performance of machine learning methods}

All machine learning methods were trained in various configurations and with several descriptors as described in methods. The prediction qualities, such as the mean $\operatorname{AUCs}(\tilde{x})$, standard deviation (sd) and coefficient of variation $\left(c v=\frac{s d}{\tilde{x}}\right)$ are shown in Table 1.

The ANNs yielded AUCs between $0.72 \pm 0.036$ (descriptor IEP) and 0.84 \pm 0.028 (descriptor hydrophobicity). According to the Wilcoxon Signed-Rank test [46] with significance level $\alpha=0.001$ the mean AUC for descriptor molecular weight was not significantly different from that obtained with descriptor hydrophobicity, while all other descriptors gave significantly lower values of mean AUC. There were no significant differences $(\alpha$ $=0.001$ ) between the mean AUCs of each descriptor with regard to the number of hidden neurons.

RFs performed consistently better than ANNs for all descriptors, reaching AUC values between $0.85 \pm 0.003$ (cleavage site prediction) and $0.93 \pm 0.001$ (hydrophobicity). Again, the best results, with only small differences, were obtained from hydrophobicity and molecular weight as descriptors. The OOB error with this descriptor was $7.59 \%$. For comparison, the best single decision tree, which was created with rpart in $\mathrm{R}$ [37], reached a pro forma AUC of 0.841 (see Table 1).

The RFs find the most important sequence positions for resistance prediction in the second half of the $\mathrm{p} 2$ sequence, especially at sequence positions 369-376 (Figure 2) in the clustalw alignment; in the wild type sequence this region corresponds to the motif QVTNSATI. The two positions 370 (V in wild type) and 372 ( $\mathrm{N}$ in wild type) have by far the highest importance in the investigated data set. This finding is in partial agreement with the findings of other workers who identified the QVT motif at positions 369-371 as important [7]. Positions 363 and 364 are not as prominent in terms of importance, although they were previously identified as crucial [47] for resistance to BVM. The apparently lower importance of these positions in the current study can be explained by the nature of our data set, which focuses on resistance mediated by baseline mutations within the $\mathrm{p} 2$ region in clinical HIV isolates.

We also trained RFs on the actual sequences, i.e. without numerical descriptors. These RFs gave OOB errors above those trained with hydrophobicities, namely of $13.55 \%$ for the $\mathrm{t}$-coffee alignment and $14.84 \%$ for the clustalw alignment. For comparison, other machine learning methods were tested as well, including Hidden Markov Models (HMMs) [48] and linear models. We tested linear support vector machines (SVMs) and logistic regression as implemented in $\mathrm{R}$ [37], and furthermore, simple perceptrons implemented in Java http:// www.heatonresearch.com/encog. All of these models performed worse compared to the RFs. The best linear model (AUC $0.826 \pm 0.008$ ) was a linear SVM using hydrophobicity as a descriptor. In Table 1 we report the 
Table 1 Area under the curve.

\begin{tabular}{|c|c|c|c|c|}
\hline method & descriptor & mean AUC & sd & cv \\
\hline \multirow[t]{5}{*}{$\overline{\mathrm{RF}}$} & hydrophobicity & 0.927 & 0.001 & 0.001 \\
\hline & molecular weight & 0.923 & 0.001 & 0.001 \\
\hline & IEP & 0.909 & 0.001 & 0.001 \\
\hline & pKa & 0.914 & 0.001 & 0.001 \\
\hline & cleavage site prediction & 0.851 & 0.003 & 0.003 \\
\hline \multirow[t]{5}{*}{$\overline{\text { ANN }}$} & hydrophobicity & 0.841 & 0.028 & 0.034 \\
\hline & molecular weight & 0.839 & 0.022 & 0.026 \\
\hline & IEP & 0.721 & 0.036 & 0.050 \\
\hline & pKa & 0.733 & 0.028 & 0.038 \\
\hline & cleavage site prediction & 0.762 & 0.036 & 0.047 \\
\hline \multirow[t]{5}{*}{ linear model } & hydrophobicity & 0.826 & 0.008 & 0.009 \\
\hline & molecular weight & 0.811 & 0.000 & 0.000 \\
\hline & IEP & 0.784 & 0.000 & 0.000 \\
\hline & pKa & 0.777 & 0.000 & 0.000 \\
\hline & cleavage site prediction & 0.803 & 0.000 & 0.000 \\
\hline \multirow[t]{5}{*}{ decision tree } & hydrophobicity & 0.815 & 0.000 & 0.000 \\
\hline & molecular weight & 0.841 & 0.000 & 0.000 \\
\hline & IEP & 0.771 & 0.000 & 0.000 \\
\hline & pKa & 0.764 & 0.000 & 0.000 \\
\hline & cleavage site prediction & 0.803 & 0.000 & 0.000 \\
\hline JRip & hydrophobicity & 0.825 & 0.000 & 0.000 \\
\hline PART & hydrophobicity & 0.890 & 0.000 & 0.000 \\
\hline Rule372 & hydrophobicity & 0.710 & 0.000 & 0.000 \\
\hline
\end{tabular}

Results of the 100-fold leave-one-out validation. The pro forma AUC values for the discrete methods (decision trees and rule based models) are just for comparison purposes. sd: standard deviation; cv: coefficient of variation.
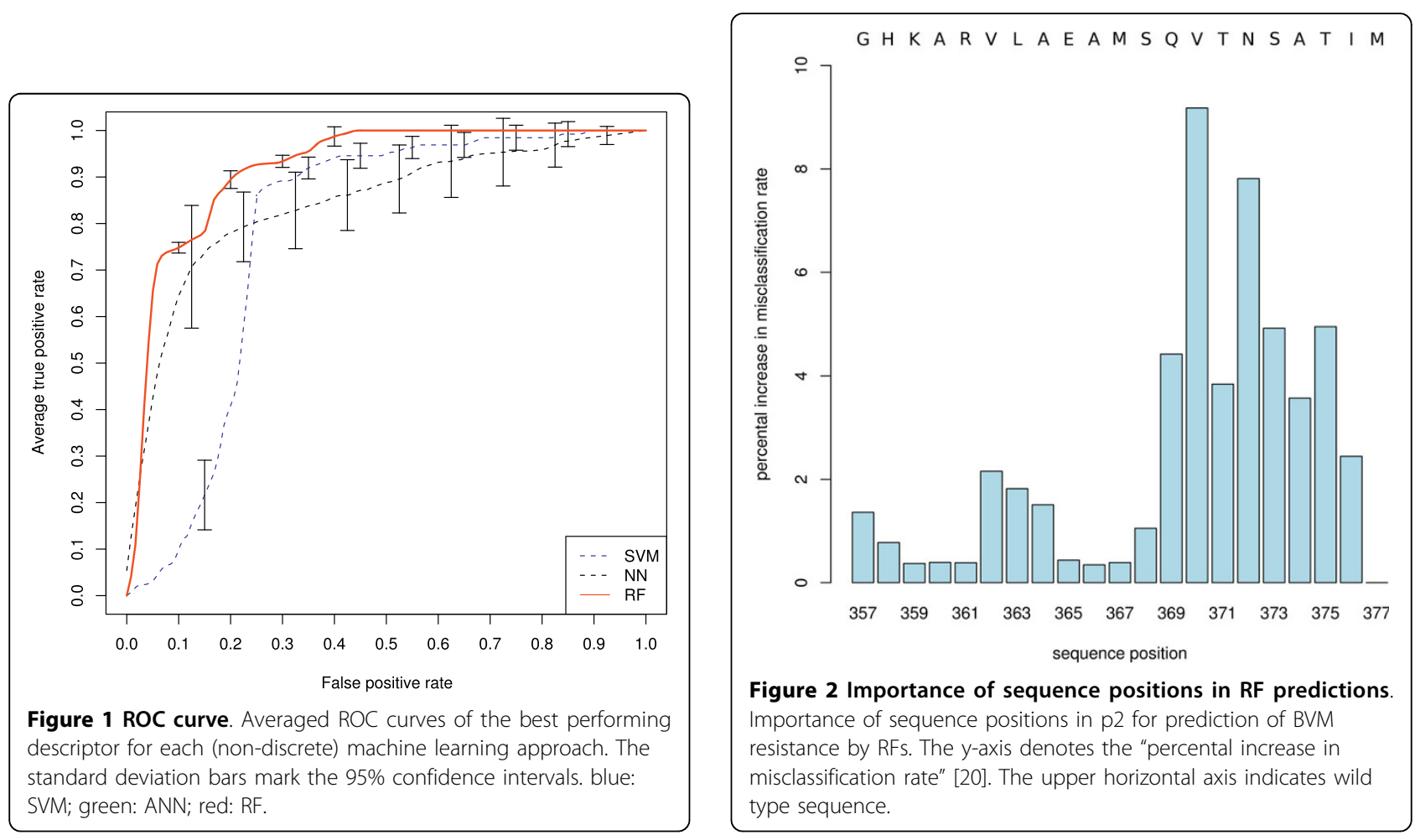
results of the best linear model for each descriptor. The HMMs were not able to classify the sequences. In Figure 1 the ROC-curves for the descriptors performing best for each (non-discrete) machine learning method are shown.

\section{Genotype-phenotype rules}

The two algorithms JRip and PART for rule extraction provided rule sets that performed well in the cross-validation with accuracies reaching almost that of RFs. Since the rules derived from the $\mathrm{t}$-coffee alignment had lower errors than those based on the clustalw alignment, we here report only the former rules.

During cross-validation JRip generated most frequently a set of three rules relating alignment positions, hydrophobicities, and BVM resistance class. Translated to amino acid residues, the rules are:

1. IF position $370 \in\{I, V\}$ AND NOT position 373 $\in\{K, R\}$ AND position $374 \in\{I, V, L, F, C, M, A\} \Rightarrow$ susceptible

2. IF position $372 \in\{K, R\}$ AND position $373 \in\{P$, $H, E, N, Q, D\} \Rightarrow$ susceptible

3. ELSE resistant

JRip reaches in the cross-validation a mean sensitivity of $77.01 \%$ at a specificity of $88.14 \%$. Dropping the first rule leads to a sensitivity of $11.76 \%$ and a specificity of 99.21\%. Dropping the second rule leads to a sensitivity of $72.54 \%$ with a corresponding specificity of $88.1 \%$.

In the cross-validation PART most frequently extracted fifteen rules (see additional file 4) with a sensitivity of $85.5 \%$ and a specificity of $93.27 \%$. Remarkably, the PART rules did take exactly those sequence positions into account that had non-zero importance in the RF analysis (see Figure 2). As suggested by the JRip and PART rules, resistance is generally caused by patterns of two or more residues. However, the importance plot (Figure 2) show that single positions may be useful indicators as well. E.g. we found that at sequence position 372 the hydrophobicity values of resistant and susceptible group clustered around two different values, 0.39 for the resistant and 0.26 for the susceptible. From this we could derive the rule (Rule372): a sequence is resistant if the hydrophobicity at 372 is closer to the mean hydrophobicity of the resistant cluster than to that of the susceptible cluster and vice versa. The rule is predictive with $52 \%$ sensitivity and $90 \%$ specificity.

\section{Structural and functional implications of resistance} mutations

After experiments [49] have excluded the classical molecular mechanism of protease inhibition, i.e. blocking of its catalytic site, there are still several molecular mechanisms for BVM action considered in the literature (for review see [1]): BVM could directly occlude the protease cleavage site ("direct" mechanisms, possibly with contact of BVM and protease), or it could stabilize a Gag structure that has to be weakened or dissolved to make the cleavage sites accessible to the protease ("indirect" mechanisms, possibly without contact of BVM and protease). Accordingly, there are several possible resistance mechanisms discussed in the literature, such as mutations that perturb the BVM binding site, that weaken the mentioned Gag structure, or that make the affected cleavage site easier digestible for the protease. A hypothetical resistance mechanism that to our knowledge so far has not been addressed is a shift of the cleavage site. We have therefore investigated associations of resistance mutations with cleavage site locations properties, as predicted computationally. In all susceptible and most resistant sequences the predicted PR cleavage sites with maximum probability were unchanged with respect to the wild type (see additional file 5): cleavage was predicted to be most probable at $P_{1}$-sites 363 and 367 in agreement with experimental findings [50], and cleavage probabilities at $\mathrm{P}_{1} 363$ were rather invariable across the data set. In a few resistant sequences cleavage sites probabilities were indeed predicted to shift (see additional file 6). Amongst these sequences we observed a tendency for the second cleavage site at $\mathrm{P}_{1}$ 367 to have lower probabilities whereas position 365 did emerge as a new possible $P_{1}$ site. However, since this occurs rather rarely, the data do not support a shift of the cleavage site as a major resistance mechanism. It is notable that in the studied data the positions 372-376 most relevant for resistance (Figure 2) lie outside the protease binding region $\mathrm{P}_{4}-\mathrm{P}_{4}$ ' for $\mathrm{P} 1$ at $363\left(\mathrm{P}_{4}{ }^{\prime} 367\right)$. Even for the internal cleavage site at $\mathrm{P}_{1} 367\left(\mathrm{P}_{4}^{\prime} 371\right)$, more than half of these important positions are outside the protease binding site. This finding is consistent with a model that allows for an "indirect" mechanism of BVM, though it cannot exclude "direct" mechanisms. In fact, mutations found in other studies closer to the cleavage sites $[47,49]$ also allow for a direct model.

A key component of an indirect mechanism is a structure within Gag that has to be weakened prior to cleavage of $\mathrm{p} 24 / \mathrm{p} 2$. A candidate structure is the $\alpha$-helix first predicted by Accola et al. [50]. We have extended secondary structure predictions to all sequences of the data set, including the wild type. All these structures were predicted as mainly $\alpha$-helical in the central part (additional file 7). This gross feature is consistent with the experimental structure by Morellet et al. [51], though the predicted helices are shorter. While in the Morellet structure the helix comprises all of the residues starting at position 358, the predicted helices comprise between seven and twelve of the 21 sequence positions and typically start at position 361 (Figure 3A). Apart from the deficiencies of the prediction method the difference 
between experiment and prediction may be due in part to the experimental conditions [51] where a substantial amount of trifluoroethanol in the solution could have led to a helix content exceeding that in the native state. The earlier work by Worthylake et al. [52] supports the view that the helix formed by $\mathrm{p} 2$ as such is not very stable. A very stable helix at the cleavage site could possibly prevent PR from cleaving, because the protease requires its substrate in an extended conformation [53]. On the other hand, recent data from electron microscopy [54] are compatible with bundles of six p2 helices stabilizing the immature matrix of the virus. In summary predictions and experiments point to a weak $\mathrm{p} 2$ helix that is stabilized by its environment.

It is remarkable that the end of the predicted helices around position 369 coincides with start of the sequence region most important for resistance (Figure 2) in our data set; in other words, the sequence positions most important for resistance in our data lie outside the predicted $\alpha$-helix in a region of unspecified secondary structure. Moreover, the resistant sequences have a tendency for shorter helices compared to susceptible sequences, as can be seen in the earlier drop of helix probability at around position 368 in Figure 3A.

We have also analyzed the confidence with which the secondary structure prediction algorithm assigns residues to a helical state. If we assume that the prediction is based on a representative sample of sequences observed as helices and non-helices, respectively, then this confidence could have a positive correlation with helix stability. A comparison of resistant and susceptible sequences with respect to mean confidence along the helix shows that resistant sequences have a tendency to lower helix confidence, and, if the assumption holds, lower helix stability (Figure 3B).

The above tendencies of resistance class, predicted helix length and confidence may reflect possible "indirect" resistance mechanisms: shorter and weaker helices
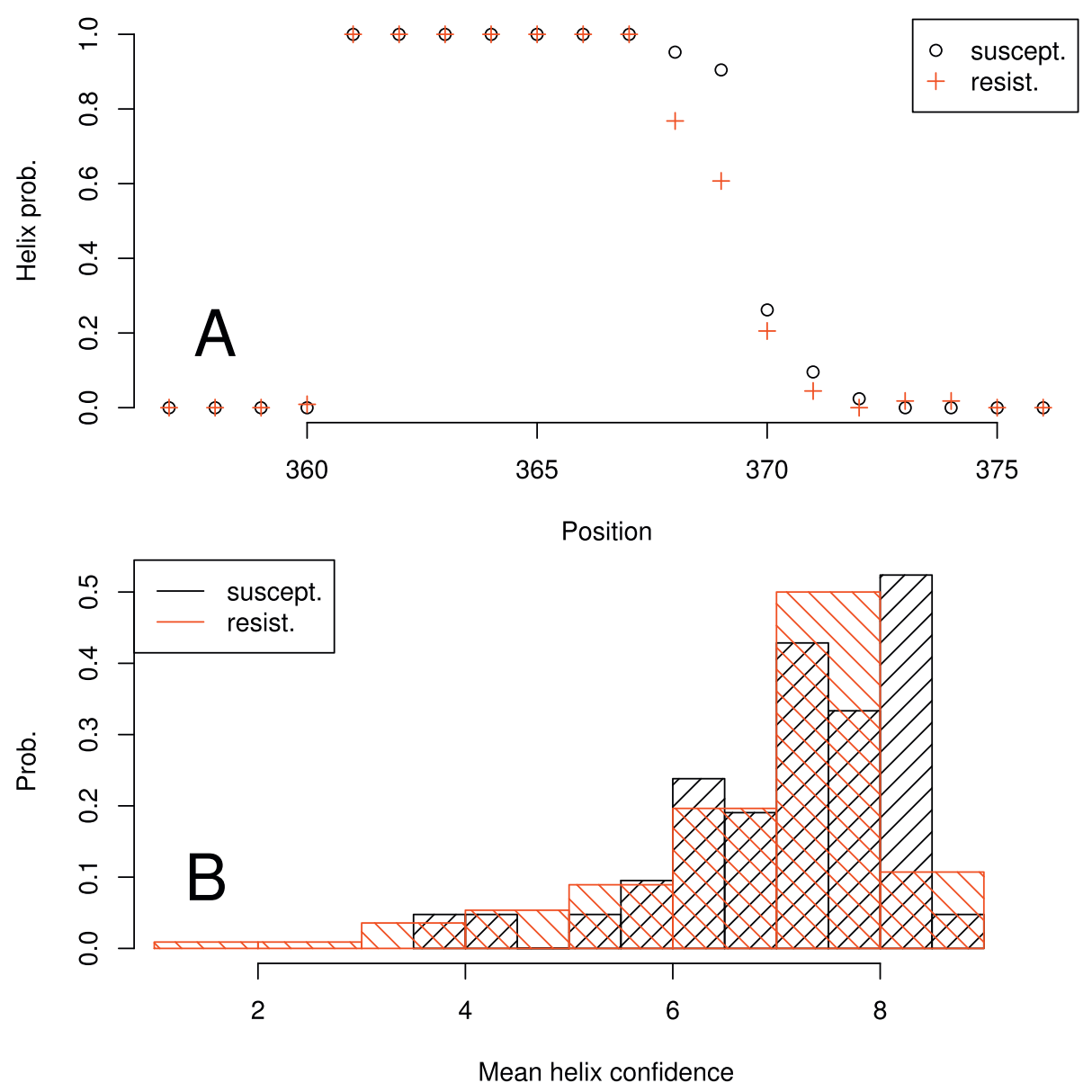

Figure 3 Helix length and confidence. Secondary structure predictions for p2 with susceptibility and resistance to BVM. A: For all p2 sequences the secondary structure was predicted by JPred [43] and then for each sequence position the helix probability (fraction of helix at this position) was computed separately for the susceptible and resistant sequences. B: Histograms of the confidence with which JPred predicts a helix (0 lowest, 9 highest). The confidence values were averaged for each sequence over all positions predicted to be helical. 
could limit the effect of BVM in several ways, e.g. by destabilizing the binding site of BVM that may lie on the six-helix bundle mentioned above, or by easing the unraveling of the remaining helix, and thus cleavage by $\mathrm{PR}$ in presence of BVM. This argument suggests to test whether helix length and helix confidence are predictive of resistance. We have therefore trained another random forest solely with the predicted helix lengths and confidences, and without reference to the detailed sequences. This random forest had an OOB error of $23 \%$, which is not as good as the errors of $8-15 \%$ reported above for random forests or rule-based methods trained on sequence information, but still much better than random guessing. This means that tuning of helicality of $\mathrm{p} 2$ could indeed be a BVM resistance mechanism.

\section{Conclusions}

BVM was the first drug of the new class of maturationinhibitors of HIV-1 that has reached phase II clinical trials. Several polymorphisms in p2 of HIV-1 hampered the sustained suppression of viral replication in these patients and conferred phenotypic resistance [7]. Since these polymorphisms were found in about $30 \%$ of treatment naïve HIV isolates and were significantly accumulated in PI resistant HIV isolates [55], genotypic resistance testing seems to be mandatory before administration of BVM.

Our analysis has shown that with the available sequences and corresponding phenotypic data it is possible to train machine learning methods that predict phenotypic resistance to BVM, mediated by baseline mutations of the $\mathrm{p} 2$ region, for unseen sequences with an error of less than $10 \%$. This result is compatible with the view that mutations in $\mathrm{p} 2$ are the main reason for BVM resistance observed in clinical isolates not responding to BVM in clinical phase I and II studies. The high classification accuracy is encouraging for personalized therapy based on genotypical testing in case BVM-like drugs will become part of the antiretroviral repertoire. With a larger, representative data set of genotype-phenotype pairs, it could become feasible to use machine learning methods not only for classification but also for regression, i.e. prediction of quantitative resistance factors.

Random forests, the method with the best classification results amongst those tested, also allowed for the identification of the sequence positions most relevant for resistance. In our data set, these sequence positions cluster in the $\mathrm{C}$-terminal half of $\mathrm{p}_{2}$, mostly outside the $\mathrm{P}_{4}-\mathrm{P}_{4}$ ' protease binding region. This is in agreement with the outcome of rule-based methods.

As judged from predicted cleavage positions, resistance mutations do usually not shift the cleavage site. Secondary structure prediction shows that resistance mutations may affect the length and strength of the $\alpha$-helix formed by at least sequence positions 371-377 and covering also the cleavage site. This hypothesis is in agreement with propositions by other workers [1] and suggests possible resistance mechanisms that also may occur in combination, e.g. (a) resistance mutations could destroy the BVM binding site that may lie in the C-terminal half of $\mathrm{p} 2$, formed by several $\mathrm{p} 2$ peptides in the six-helix bundle suggested by Wright et al. [54]; (b) resistance mutations could weaken the $\alpha$-helix in $\mathrm{p} 2$, and thus, the six-helix bundle in the immature virus. This could ease unraveling of the helix prior to cleavage by PR, and hence, may functionally outweigh a stabilizing effect of BVM on the helix bundle.

Additional file 1: Data set. The sequences used in this study.
Click here for file
[http://www.biomedcentral.com/content/supplementary/1471-2105-11-
37-S1.XLS]
Additional file 2: MSA of the sequences with clustalw. Multiple
sequence alignment of the sequences with clustalw [23].
Click here for file
[http://www.biomedcentral.com/content/supplementary/1471-2105-11-
37-S2.TXT]
Additional file 3: MSA of the sequences with t-coffee. Multiple
sequence alignment of the sequences with t-coffee [24].
Click here for file
[http://www.biomedcentral.com/content/supplementary/1471-2105-11-
37-S3.TXT]
Additional file 4: Plots and rules. Variance plots and prediction rules.
Click here for file
[http://www.biomedcentral.com/content/supplementary/1471-2105-11-
37-S4.PDF]
Additional file 5: Cleavage site predictions. Predictions are made with
HIVcleave [31].
Click here for file
[http://www.biomedcentral.com/content/supplementary/1471-2105-11-
37-S5.XLS ]
Additional file 6: Shifted cleavage site probabilities. Probable HIV-
protease cleavage sites are shown in bold [31]. The value represents the
probability of protease cleavage.
Click here for file
[http://www.biomedcentral.com/content/supplementary/1471-2105-11-
37-S6.XLS ]
Additional file 7: Secondary structure predictions. Predictions are
made with JPred [43].
Click here for file
[http://www.biomedcentral.com/content/supplementary/1471-2105-11-
37-S7.XLS ]

\section{Acknowledgements}

The authors thank J. Nikolaj Dybowski for the assistance and helpful discussions. We also thank Dr. Nelly Morellet (Universitè Paris-Descartes, Paris, France) for the p24/p2 structural model. This work was supported by the Deutsche Forschungsgemeinschaft (SFB/Transregio 60).

\section{Author details}

'Department of Bioinformatics, Center of Medical Biotechnology, University of Duisburg-Essen, Universitaetsstr. 2, 45117 Essen, Germany. ${ }^{2}$ Institute of Virology, University of Cologne, Fuerst-Pueckler-Str. 56, 50935 Cologne, Germany. 


\section{Authors' contributions}

All authors have jointly developed the research concept and collaborated on the writing of the manuscript. $\mathrm{DH}^{*}$ has carried out computational analyses and drafted the manuscript. JV has initiated the study and revised the manuscript. DH has interpreted results and revised the manuscript. All authors read and approved the final manuscript.

Received: 24 August 2009

Accepted: 20 January 2010 Published: 20 January 2010

\section{References}

1. Salzwedel K, Martin D, Sakalian M: Maturation inhibitors: a new therapeutic class targets the virus structure. AIDS Rev 2007, 9:162-172.

2. Adamson CS, Ablan SD, Boeras I, Goila-Gaur R, Soheilian F, Nagashima K, Li F, Salzwedel K, Sakalian M, Wild CT, Freed EO: In vitro resistance to the human immunodeficiency virus type 1 maturation inhibitor PA-457 (Bevirimat). J Virol 2006, 80(22):10957-10971.

3. Li F, Zoumplis D, Matallana C, Kilgore N, Reddick M, Yunus A, Adamson C, Salzwedel K, Martin D, Allaway G, Freed E, Wild C: Determinants of activity of the HIV-1 maturation inhibitor PA-457. Virology 2006, 356:217-24.

4. Adamson CS, Waki K, Ablan SD, Salzwedel K, Freed EO: Impact of human immunodeficiency virus type 1 resistance to protease inhibitors on evolution of resistance to the maturation inhibitor bevirimat (PA-457). $J$ Virol 2009, 83(10):4884-4894.

5. Margot N, Gibbs C, Miller M: Phenotypic susceptibility to Bevirimat among HIV-infected patient isolates without prior exposure to Bevirimat. Proceedings of the 16th Conference on Retroviruses and Opportunistic Infections, Montreal, Canada 2009.

6. Salzwedel K, Harmy F, Louvel S, Sakalian M, Reddick M, Finnegan C, Martin D, McCallister S, Klimkait T, Allaway G: Susceptibility of diverse HIV1 patient isolates to the maturation inhibitor, Bevirimat (MPC-4326), is determined by clade-specific polymorphisms in Gag CA-SP1. Proceedings of the 16th Conference on Retroviruses and Opportunistic Infections, Montreal, Canada 2009.

7. Baelen KV, Salzwedel K, Rondelez E, Eygen W, Vos SD, Verheyen A, Steegen K, Verlinden Y, Allaway GP, Stuyver L: Susceptibility of human immunodeficiency virus type 1 to the maturation inhibitor bevirimat is modulated by baseline polymorphisms in Gag spacer peptide 1 . Antimicrob Agents Chemother 2009, 53:2185-2188.

8. McCallister S, Lalezari J, Richmond G, Thompson M, Harrigan R, Martin D, Salzwedel K, Allaway G: HIV-1 Gag polymorphisms determine treatment response to bevirimat (PA-457). Antivir Ther 2008, 13(Suppl 3):A10.

9. Lathrop R, Steffen N, Raphael M, Deeds-Rubin S, Pazzani M, Cimoch P, See D, Tilles J: Knowledge-based avoidance of drug-resistant HIV mutants. Al MAGAZINE 1999, 20(1):13-25.

10. Sevin AD, DeGruttola V, Nijhuis M, Schapiro JM, Foulkes AS, Para MF, Boucher CAB: Methods for Investigation of the Relationship between Drug-Susceptibility Phenotype and Human Immunodeficiency Virus Type 1 Genotype with Applications to AIDS Clinical Trials Group 333. J Infect Dis 2000, 182:59-67.

11. Beerenwinkel N, Schmidt B, Walter H, Kaiser R, Lengauer T, Hoffmann D, Korn K, Selbig J: Diversity and complexity of HIV-1 drug resistance: a bioinformatics approach to predicting phenotype from genotype. Proc Natl Acad Sci USA 2002, 99(12):8271-8276.

12. Beerenwinkel N, Schmidt B, Walter H, Kaiser R, Lengauer T, Hoffmann D, Korn K, Selbig J: Geno2pheno: Interpreting Genotypic HIV Drug Resistance Tests. IEEE Intelligent Systems 2001, 16:35-41.

13. Murray RJ, Lewis Fl, Miller MD, Brown AJ: Genetic basis of variation in tenofovir drug susceptibility in HIV-1. AIDS 2008, 22(10):1113-23.

14. Resch W, Hoffman N, Swanstrom R: Improved success of phenotype prediction of the human immunodeficiency virus type 1 from envelope variable loop 3 sequence using neural networks. Virology 2001, 288:51-62.

15. Draghici S, Potter RB: Predicting HIV drug resistance with neural networks. Bioinformatics 2003, 19:98-107.

16. Wang $D$, Larder $B$ : Enhanced prediction of lopinavir resistance from genotype by use of artificial neural networks. J Infect Dis 2003, 188(5):653-660.

17. King R, Feng C, Sutherland A: Comparison of classification algorithms on large real-world problems. Applied Artificial Intelligence 1995, 9(3):259-287.
18. Tzafestas S, Dalianis PJ, Anthopoulos G: On the overtraining phenomenon of backpropagation neural networks. Mathematics and computers in simulation 1996, 40:505-663.

19. Banfield RE, Hall LO, Bowyer KW, Kegelmeyer WP: A comparison of decision tree ensemble creation techniques. IEEE Transactions on Pattern Analysis and Machine Intelligence 2007, 29(1):173-180.

20. Breiman L: Random Forests. Machine Learning 2001, 45:5-32.

21. Kingston J: Rule-based expert systems and beyond: an overview. British Association of Accountants' Conference 1987.

22. Witten $\mathbb{I H}$, Frank E: Data Mining. Morgan Kauffmann 2000.

23. Thompson J, Higgins D, Gibson T: CLUSTAL W: improving the sensitivity of progressive multiple sequence alignment through sequence weighting, position-specific gap penalties and weight matrix choice. Nucleic Acids Res 1994, 22:4673-4680.

24. Notredame C, Higgins DG, Heringa J: T-Coffee: A novel method for fast and accurate multiple sequence alignment. J Mol Biol 2000, 302:205-217.

25. Edgar RC: MUSCLE: a multiple sequence alignment method with reduced time and space complexity. BMC Bioinformatics 2004, 5:113.

26. Löytynoja A, Goldman N: An algorithm for progressive multiple alignment of sequences with insertions. Proc Natl Acad Sci USA 2005, 102(30):10557-10562

27. Ong S, Lin H, Chen Y, Li Z, Cao Z: Efficacy of different protein descriptors in predicting protein functional families. BMC Bioinformatics 2007, 8:300.

28. Kernytsky A, Rost B: Using genetic algorithms to select most predictive protein features. Proteins 2009, 75:75-88.

29. Nanni L, Lumini A: Using ensembles of classifiers for predicting HIV protease cleavage sites in proteins. Amino Acids 2009, 36:409-416.

30. Kyte J, Doolittle R: A simple method for displaying the hydropathic character of a protein. J Mol Biol 1982, 157:105-132.

31. Shen HB, Chou KC: HIVcleave: a web-server for predicting human immunodeficiency virus protease cleavage sites in proteins. Analytical Biochemistry 2008, 375:388-390.

32. Riedmiller $\mathrm{M}$, Braun $\mathrm{H}$ : A direct adaptive method for faster backpropagation learning: The Rprop algorithm. Proceedings of the IEEE International Conference on Neural Networks 1993, 586-591.

33. Borschbach M, Hauke S, Pyka M, Heider D: Opportunities and limitations of a principal component analysis optimized machine learning approach for the identification and classification of cancer involved proteins. Communications of the SIWN 2009, 6:85-89.

34. Heider D, Appelmann J, Bayro T, Dreckmann W, Held A, Winkler J, Barnekow A, Borschbach M: A computational approach for the identification of small GTPases based on preprocessed amino acid sequences. Technology in Cancer Research and Treatment 2009, 8(5):333-342.

35. Nguyen D, Widrow B: Improving the learning speed of 2-layer neural networks by choosing initial values of the adaptive weights. Proceedings of Intl Joint Conf on Neural Networks 1990, 21-26.

36. Punta M, Rost B: Neural networks predict protein structure and function Humana Press, Berlin, Germany 2008 chap. Artificial Neural Networks: Methods and Protocols.

37. R Development Core Team: R: A Language and Environment for Statistical Computing R Foundation for Statistical Computing, Vienna, Austria 2006http://www.R-project.org, ISBN 3-900051-07-0.

38. Cohen WW: Fast effective rule induction. Proceedings of the 12th International Conference on Machine Learning Prieditis A, Russell S 1995, 115-123.

39. Frank $E$, Witten $I H$ : Generating accurate rule sets without global optimization. Machine Learning: Proceedings of the Fifteenth International Conference Shavlik J 1998.

40. Cawley GC: Leave-One-Out Cross-Validation Based Model Selection Criteria for Weighted LS-SVMs. Proceedings of the IEEE World Congress on Computational Intelligence 2006.

41. Fawcett T: An introduction to ROC analysis. Pattern Recognition Letters 2006, 27:861-874.

42. $\quad$ Sing $T$, Sander O, Beerenwinkel N, Lengauer T: ROCR: visualizing classifier performance in R. Bioinformatics 2005, 21(20):3940-3941.

43. Cole C, Barber JD, Barton GJ: The Jpred 3 secondary structure prediction server. Nucleic Acids Res 2008, 36:W197-201.

44. Chou KC, Tomasselli AG, Reardon IM, Heinrikson RL: Predicting human immunodeficiency virus protease cleavage sites in proteins by a discriminant function method. Proteins 1996, 24:51-72. 
45. Wilcoxon F: Individual comparisons by ranking methods. Biometrics 1945, 1:80-83.

46. Demsar J: Statistical comparisons of classifiers over multiple data sets. Journal of Machine Learning Research 2006, 7:1-30.

47. Zhou J, Chen $\mathrm{CH}$, Aiken C: Human immunodeficiency virus type 1 resistance to the small molecule maturation inhibitor $3-0-\left(3^{\prime}, 3^{\prime}-\right.$ dimethylsuccinyl)-betulinic acid is conferred by a variety of single amino acid substitutions at the CA-SP1 cleavage site in Gag. J Virol 2006, 80(24):12095-101.

48. Eddy SR: Profile hidden Markov models. Bioinformatics 1998, 14(9):755-63.

49. Li F, Goila-Gaur R, Salzwedel K, Kilgore NR, Reddick M, Matallana C, Castillo A, Zoumplis D, Martin DE, Orenstein JM, Allaway GP, Freed EO, Wild CT: PA-457: a potent HIV inhibitor that disrupts core condensation by targeting a late step in Gag processing. Proc Natl Acad Sci USA 2003, 100(23):13555-60.

50. Accola MA, Höglund S, Göttlinger HG: A putative alpha-helical structure which overlaps the capsid-p2 boundary in the human immunodeficiency virus type $1 \mathrm{Gag}$ precursor is crucial for viral particle assembly. J Virol 1998, 72:2072-2078.

51. Morellet N, Druillennec $S$, Lenoir C, Bouaziz S, Roques B: Helical structure determined by NMR of the HIV-1 (345-392)Gag sequence, surrounding p2: Implications for particle assembly and RNA packaging. Protein Science 2004, 14:375-386.

52. Worthylake DK, Wang H, Yoo S, Sundquist WI, Hill CP: Structures of the HIV-1 capsid protein dimerization domain at 2.6 A resolution. Acta Crystallogr D Biol Crystallogr 1999, 55:85-92.

53. Miller M, Schneider J, Sathyanarayana BK, Toth MV, Marshall GR, Clawson L, Selk L, Kent SB, Wlodawer A: Structure of complex of synthetic HIV-1 protease with a substrate-based inhibitor at $2.3 \mathrm{~A}$ resolution. Science 1989, 246(4934):1149-52.

54. Wright ER, Schooler JB, Ding HJ, Kieffer C, Fillmore C, Sundquist WI, Jensen GJ: Electron cryotomography of immature HIV-1 virions reveals the structure of the CA and SP1 Gag shells. EMBO J 2007, 26(8):2218-26.

55. Verheyen J, Verhofstede C, Knops E, Vandekerckhove L, Fun A, Brunen D, Dauwe K, Wensing A, Pfister H, Kaiser R, Nijhuis M: High prevalence of bevirimat resistance mutations in protease inhibitor-resistant HIV isolates. AIDS 2009.

Submit your next manuscript to BioMed Central and take full advantage of:

- Convenient online submission

- Thorough peer review

- No space constraints or color figure charges

- Immediate publication on acceptance

- Inclusion in PubMed, CAS, Scopus and Google Scholar

- Research which is freely available for redistribution 